\title{
Caracterização de solos de duas formações de restinga e sua influência na constituição química foliar de Passiflora mucronata Lam. (Passifloraceae) e Canavalia rosea (Sw.) DC. (Fabaceae) $)^{1}$
}

\author{
Jehová Lourenço Junior ${ }^{2,4}$ e Geraldo Rogério Faustini Cuzzuol ${ }^{3}$
}

Recebido em 16/10/2007. Aceito em 26/05/2008

\begin{abstract}
RESUMO - (Caracterização de solos de duas formações de restinga e sua influência na constituição química foliar de Passiflora mucronata Lam. (Passifloraceae) e Canavalia rosea (Sw.) DC. (Fabaceae)). As hipóteses sobre o posicionamento das formações de restinga são diversas sendo o gradiente salino e a fertilidade do solo as mais difundidas. Buscando elucidar essas questões, foram analisadas as propriedades dos solos das formações psamófila-reptante (FPR) e Palmae (FP) bem como a constituição química foliar de $C$. rosea (Sw.) DC. restrita à FPR e P. mucronata Lam., na FP. O solo da FP destacou-se pela maior fertilidade. Maiores teores dos metais Fe, Zn e ISNa foram encontrados em solos da FPR. Não foi detectada a presença de Al nas duas formações. O pH dos solos é alcalino sem diferenças entre as duas formações. Os elementos químicos $\mathrm{P}, \mathrm{S}, \mathrm{Cu}$ e $\mathrm{Zn}$ acumulam-se em maiores concentrações em folhas de $P$. mucronata. C. rosea destacou-se pelos maiores teores foliares de N, Fe e B. Essa Fabaceae mostrou valores superiores do fator de concentração para o $\mathrm{Ca}, \mathrm{Mg}, \mathrm{Fe}, \mathrm{Cu}$ e B enquanto que . mucronata, para o $\mathrm{P}$ e Zn. Os resultados obtidos sugerem que as características pedológicas têm papel chave no estabelecimento de $C$. rosea na FPR e de P. mucronata na FP.
\end{abstract}

Palavras-chave: distribuição espacial, ecofisiologia, nutrição mineral, zonação

ABSTRACT - (Soil characterization of two restinga formations and its influence on leaf chemistry in Passiflora mucronata Lam. (Passifloraceae) and Canavalia rosea (Sw.) DC. (Fabaceae)). Hypotheses as to the position of restinga formations are many, the most widespread being the salinity gradient and soil fertility. In order to elucidate these questions, soil properties of the creeping psammophyte $(\mathrm{CPF})$ and Palmae formations (PF) as well as leaf chemistry of C. rosea (Sw.) DC. restricted to the former and P. mucronata Lam. in the latter were analyzed. The PF soil was more fertile. Higher content of Fe, Zn and ISNa was found in CPF soil. The presence of Al in these two formations was not detected. Soil $\mathrm{pH}$ is alkaline, with no difference found between the two formations. The chemical elements $\mathrm{P}$, $\mathrm{S}$, $\mathrm{Cu}$ and $\mathrm{Zn}$ accumulate in greater concentrations in $P$. mucronata leaves. $C$. rosea was distinguished by a higher leaf content of $\mathrm{N}, \mathrm{Fe}$ and B. This legume showed greater factor of concentration values for $\mathrm{Ca}, \mathrm{Mg}, \mathrm{Fe}, \mathrm{Cu}$ and $\mathrm{B}$ whereas . mucronata, for $\mathrm{P}$ and $\mathrm{Zn}$. The results suggest that soil characteristics play a major role in the development of $C$. rosea in the $\mathrm{CPF}$ and of $P$. mucronata in the PF.

Key words: ecophysiology, mineral nutrition, spatial distribution, zonation

\section{Introdução}

A restinga é um ecossistema típico da costa atlântica brasileira (Araujo 1992) originado pelo acúmulo de sedimentos cristalinos e da transgressão marinha no Quartenário (Suguio \& Martin 1990), representando cerca de $70 \%$ do litoral brasileiro (Araujo 1992). Dos 75.000 ha da vegetação original restam apenas 10.000 ha da restinga do Espírito Santo devido à especulação imobiliária, expansão das áreas de agropecuária e invasão de espécies exóticas devido às alterações antrópicas (Scherer et al. 2005). Embora a floresta de restinga esteja estabelecida em solos pobres em nutrientes, sua vegetação está bem adaptada aos ambientes oligotróficos demonstrando elevada eficiência na utilização dos nutrientes minerais (Moraes et al. 1999).

As restingas do Espírito Santo se destacam pela riqueza de sua flora abrigando espécies e comunidades vegetais com características estruturais bastante distintas (Pereira 1990a). Na restinga do Parque Estadual Paulo Cezar Vinha, em Guarapari, Espírito Santo, são identificadas doze formações (Pereira 1990a; b) bem estruturadas (halófila, psamófila-reptante, pós-praia, Palmae, mata de Myrtaceae, mata seca, aberta de Clusia, brejo herbáceo, floresta periodicamente inundada, floresta permanentemente inundada e formação de Ericaceae)

\footnotetext{
1 Parte da Dissertação de Mestrado do primeiro Autor

2 Universidade Federal do Espírito Santo, Programa de Pós-Graduação em Biologia Vegetal (PPGBV), Av. Fernando Ferrrari 514, Campus Universitário Alaor Queiroz de Araújo, 29075-910 Vitória, ES, Brasil

3 Universidade Federal do Espírito Santo, PPGBV, Departamento de Ciências Biológicas/CCHN

4 Autor para correspondência: jehovajr@yahoo.com.br
} 
seguindo um gradiente de distribuição, complexidade e abundância de espécies no sentido da praia para o interior do continente (Assis et al. 2004; Pereira 1990a,b).

A formação psamófila-reptante congrega plantas em sua maioria estoloníferas, dentre as quais Ipomoea pescapre (L.) Sweet., Canavalia rosea (Sw.) DC. e Sporobulus virginicus Kunth. A formação Palmae possui uma maior diversidade de espécies quando comparada à formação psamófila-reptante, e é dominada pela palmeira anã Allagoptera arenaria (Gomes) O. Ktze (Pereira $1990 \mathrm{a} ;$ b). Nessa mesma formação, ainda são encontradas Passiflora mucronata Lam., Schinus terebinthifolius Raddi, Cereus fernambucensis Lem. e Sophora tomentosa L. (Pereira 1990a; b; Fabris 1995).

A zonação das espécies em comunidades de restinga é influenciada pelos fatores edáficos integrados com as relações nutricionais do sistema (Reinert et al. 1997) sendo que a topografia (Cordeiro 1998), a salsugem, a salinidade do solo e o movimento da areia são os mais citados (Henriques et al. 1986; Cogliatti-Carvalho et al. 2001). Além da zonação e da composição florística, outros fatores podem atuar na distribuição da vegetação e caracterização da comunidade, como a concentração de nutrientes no solo (Menezes \& Araujo 2000). De acordo com Jefferies \& Davy (1979), as diferentes formações ao longo da costa marinha indicariam os diferentes graus de resistência ao sal, o que poderia ter gerado a existência de ecótipos. Devido à proximidade ao mar, C. rosea está sob constante influência da salinidade, sobretudo nos períodos de maré alta quando as ondas adentram a formação psamófila-reptante, expondo $C$. rosea ao contato direto com a água salgada (Jefferies \& Davy 1979). Por este fato, essa espécie tem sido descrita como tolerante à salsugem que seria, hipoteticamente, o principal fator de seu posicionamento, enquanto que espécies como P. mucronata ocupariam, preferencialmente, regiões mais afastadas da linha da costa sob menor impacto da salinidade.

Além da salinidade, o padrão de distribuição de plantas em formações costeiras também tem sido relacionado à influência das propriedades físico-químicas do sedimento (Cuzzuol \& Campos 2001; Bernini et al. 2006) ficando a distribuição geográfica de plantas e composição de florestas tropicais brasileiras sujeitas às relações nutricionais do solo (Espírito Santo et al. 2002; Carvalho et al. 2005; Rodrigues et al. 2007).

Kraya \& Jarenkowb (2003) sugerem a existência de um gradiente de fertilidade para o solo de restinga levando ao aumento da complexidade estrutural da vegetação no sentido praia-continente. Dessa forma, o gradiente de fertilidade de solos e as exigências nutricionais específicas podem ser fatores determinantes no posicionamento de $P$. mucronata na formação psamófila-reptante e de C. rosea na formação Palmae da restinga do Parque Estadual Paulo César Vinha, em Guarapari.

A distribuição espacial das formações vegetais permite deduzir as preferências ecológicas das espécies nativas fundamentais para o manejo e revegetação das restingas tão severamente impactadas nas últimas três décadas. Informações desse tipo são desejáveis para a compreensão da dinâmica da distribuição espacial em florestas tropicais costeiras (Cuzzuol \& Campos 2001). $\mathrm{O}$ presente estudo tem objetivo de caracterizar as propriedades físico-químicas dos solos da formação psamófila-reptante (FPR) e da formação Palmae (FP) identificando suas possíveis interações com a composição química foliar de C. rosea, restrita à FPR, e de P. mucronata na FP da restinga do Parque Estadual Paulo César Vinha.

\section{Material e métodos}

$\mathrm{O}$ estudo foi desenvolvido entre agosto/2005 a abril/2006 na restinga do Parque Estadual Paulo César Vinha (PEPCV) localizado em planície litorânea de aproximadamente 1.500 ha em Setiba, município de Guarapari, Espírito Santo (20³3'88'S e $40^{\circ} 23^{\prime}$ $\left.40^{\circ} 26^{\prime} \mathrm{W}\right)$. O clima da região é do tipo Aw tropical, com verão quente e chuvoso e inverso seco apresentando temperatura média anual de $24{ }^{\circ} \mathrm{C}$, precipitação média anual de $900 \mathrm{~mm}$ e umidade relativa média anual de $80 \%$ no período investigado (Instituto Estadual do Meio Ambiental 2007). O sedimento do trecho estudado, bem como de outras formações dessa unidade de conservação, é predominantemente arenoso (Pereira 1990a;b; Thomaz \& Monteiro 1991; Assis et al. 2004), pobre em argilas e matéria orgânica, baixa capacidade de reter água e nutrientes e por uma grande proporção da entrada anual de nutrientes sob a forma de salsugem (Lacerda 1984).

Os materiais de estudo foram os solos da formação Palmae e da formação psamófila-reptante e os nutrientes foliares de plantas de P. mucronata e de C. rosea (Fig. 1). Foram coletadas três amostras de solos, das duas formações, em cinco níveis de profundidade (0-10, 10-20, 20-30, 30-40 e 40-50 cm). Foram coletadas folhas adultas e sadias do quarto ou quinto nó de cinco plantas de $P$. mucronata e de cinco estolões de $C$. rosea no período entre 7:00 e 9:00 h conforme recomendação de Costa et al. (2005). Amostras de folhas e solos foram submetidas às análises físico-químicas no Instituto Capixaba de Pesquisas e Extensão Rural (Incaper).

As amostras passaram por processo de secagem em estufa com ventilação forçada sob uma temperatura máxima de $40^{\circ} \mathrm{C}$. A determinação do $\mathrm{pH}$ do solo foi feita 


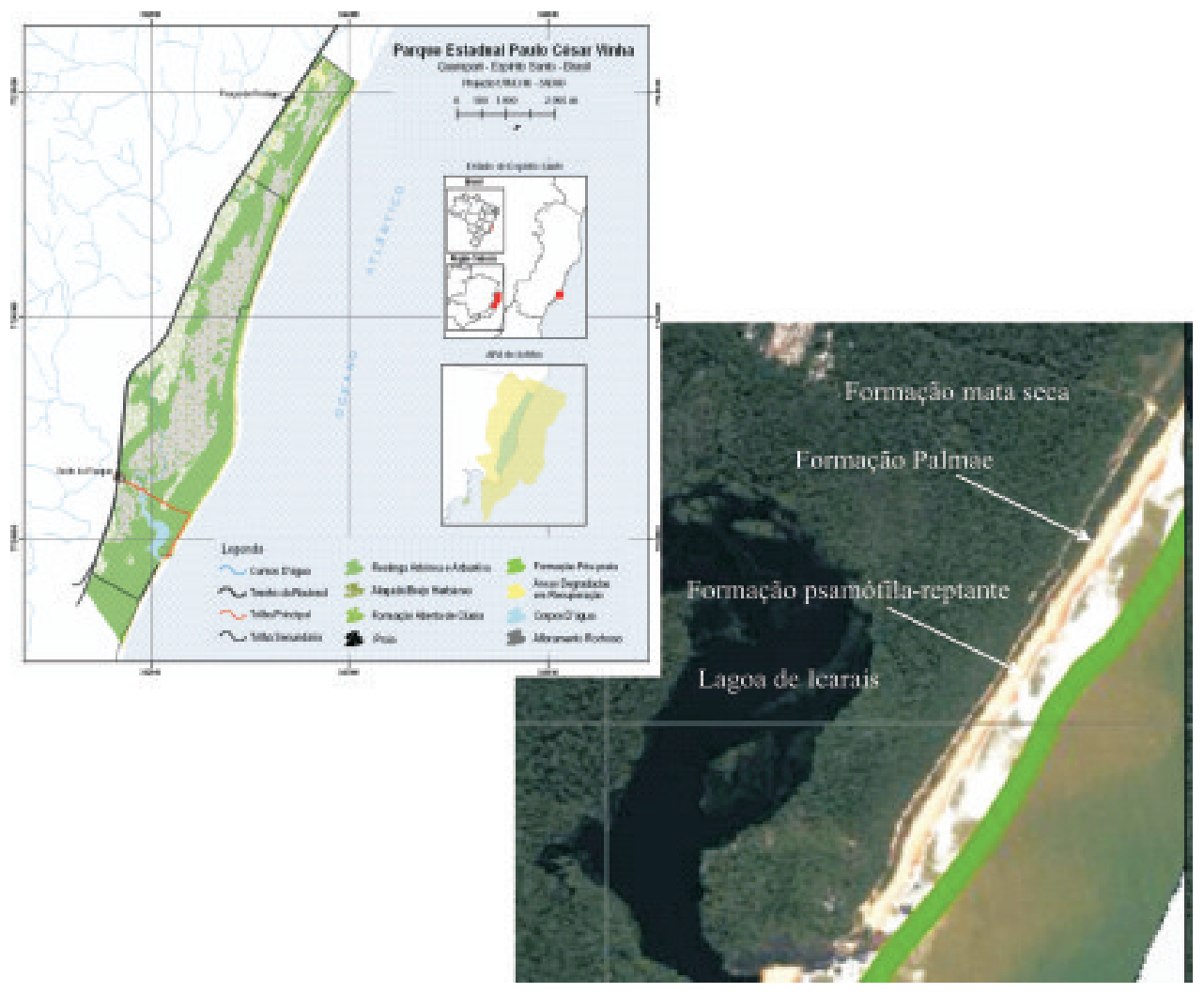

Figura 1. Caracterização geral do Parque Estadual Paulo César Vinha, (fotografia aérea) com a delimitação do Parque e da Área de Proteção Ambiental (primeira faixa à esquerda da fotografia aérea - faixa verde). Dados e imagens fornecidos pela Subgerência Geomática (SUGEO) do Instituto Estadual de Meio Ambiente (IEMA 2005). As setas indicam a localização de Canavalia rosea (Sw.) DC. na formação psamófilareptante (FPR) e de Passiflora mucronata Lam. na formação Palmae (FP).

em água com $\mathrm{KCl}$ e $\mathrm{CaCl}_{2}$, numa relação de 1:2,5. A matéria orgânica $(\mathrm{MO})$ do solo foi determinada através da oxidação por $\mathrm{Na}_{2} \mathrm{Cr}_{2} \mathrm{O}_{7} 4 \mathrm{~N}$ e $\mathrm{H}_{2} \mathrm{SO}_{4} 10 \mathrm{~N}$. Para a extração de $\mathrm{P}, \mathrm{Na}, \mathrm{K}, \mathrm{Fe}, \mathrm{Zn}, \mathrm{Mn}$ e $\mathrm{Cu}$, utilizou-se o extrator Mehlich 1. Os elementos $\mathrm{Ca}, \mathrm{Mg}$ e Al foram extraídos com o extrator $\mathrm{KCl}$ a $1 \mathrm{M}$. A determinação da acidez potencial $(\mathrm{H}+\mathrm{AL})$ foi executada através da extração pelo método SMP. A extração do B foi realizada em água quente e o $\mathrm{S}$, em solução de fosfato monocálcico em ácido acético. A granulometria do sedimento foi determinada pelo método do densímetro. As classificações texturais seguiram a metodologia de Suguio (1973), baseadas nos percentuais de areia, silte e argila. A matéria orgânica (fração $<2 \mathrm{~mm}$ ) foi determinada pelo método indireto (digestão úmida com dicromato de potássio e ácido sulfúrico). A extração das frações móveis de P, K, Fe, Mn, Zn e Cu foi realizada com solução de $\mathrm{HCl} 0,05 \mathrm{~N}+\mathrm{H} 2 \mathrm{SO} 40,025 \mathrm{~N}$, na relação solo:extrator 1:5. A determinação do $K$ foi feita por Fotometria de Chama, do P por Colorimetria $(725 \mathrm{~nm})$ e dos demais elementos, por espectrofotometria de absorção atômica convencional (EAA). O Ca e o $\mathrm{Mg}$ trocáveis foram extraídos com $\mathrm{KCl} 1 \mathrm{~N}$ e determinados pelo método complexométrico com o emprego de EDTA (titulometria). Os elementos químicos do sedimento foram determinados para a fração $<2 \mathrm{~mm}$, de acordo com a metodologia descrita por Bataglia et al. (1983) e EMBRAPA (1997). Os teores dos nutrientes foliares foram determinados segundo metodologia descrita em Sarruge \& Haag (1974) e Silva (1981). O nitrogênio total foi determinado pela digestão Microkjeldahl. Para a extração de $\mathrm{Ca}, \mathrm{Cu}, \mathrm{Fe}, \mathrm{K}, \mathrm{Mg}, \mathrm{Mn}, \mathrm{P}, \mathrm{S}$ e Zn utilizou-se digestão nítrico-perclórica. A determinação do fósforo total foi feita por Colorimetria $(725 \mathrm{~nm})$ e os demais elementos por EAA. Após digestão, o enxofre foi quantificado por determinação gravimétrica, que se baseia na precipitação do enxofre pelo cloreto de bário, na forma de sulfato de bário. A concentração foliar foi dividida pela concentração do sedimento para determinar o fator de concentração (FC) (cf. Salisbury \& Ross 1992). Os dados foram submetidos à análise de variância sendo a diferença mínima significativa determinada pelo Teste de Tukey em nível de 5\% de probabilidade utilizando o programa ASSISTAT.

\section{Resultados}

De um modo geral, as frações superficiais de solo das duas formações foram ricas em nutrientes (Tab. 1). O solo da formação Palmae (FP) apresentou maiores 
Tabela 1. Valores médios de macronutrientes $\left(\mathrm{g} \cdot \mathrm{kg}^{-1}\right)$ e micronutrientes $\left(\mathrm{mg} \cdot \mathrm{kg}^{-1}\right)$ da rizosfera de Passiflora mucronata Lam. e Canavalia rosea (Sw.) DC. na formação Palmae e psamófila-reptante, respectivamente, da restinga do Parque Estadual Paulo César Vinha, Guarapari, ES, Brasil. Nas linhas, as letras minúsculas comparam as médias dentro de profundidade e as letras maiúsculas comparam as médias entre as formações pelo teste de Duncan $(P<0,05 \%, \mathrm{n}=3)$.

\begin{tabular}{|c|c|c|c|c|c|c|c|c|c|c|}
\hline \multirow{3}{*}{$\begin{array}{l}\text { Nutrientes } \\
\left(\mathrm{mg} \cdot \mathrm{kg}^{-1}\right)\end{array}$} & \multicolumn{5}{|c|}{ Formação Palmae } & \multicolumn{5}{|c|}{ Formação psamófila-reptante } \\
\hline & \multicolumn{5}{|c|}{ Profundidade $(\mathrm{cm})$} & \multicolumn{5}{|c|}{ Profundidade $(\mathrm{cm})$} \\
\hline & 10 & 20 & 30 & 40 & 50 & 10 & 20 & 30 & 40 & 50 \\
\hline $\mathrm{P}$ & $25,3 \mathrm{bcA}$ & $19,7 \mathrm{bAa}$ & $16,4 \mathrm{abAa}$ & $15,5 \mathrm{aA}$ & $14,3 \mathrm{aA}$ & $24,6 \mathrm{bA}$ & $18,2 \mathrm{aA}$ & $16,6 \mathrm{aA}$ & 15,5 aA & $16,1 \mathrm{aB}$ \\
\hline $\mathrm{K}$ & $34,6 \mathrm{dA}$ & $14,3 \mathrm{bcA}$ & $10 \mathrm{bA}$ & $9,3 \mathrm{abA}$ & $8,6 \mathrm{aA}$ & $20,3 \mathrm{cB}$ & $12,6 \mathrm{bA}$ & $11,6 \mathrm{abA}$ & $10 \mathrm{aAa}$ & 9,3 aA \\
\hline $\mathrm{Ca}$ & $2304 \mathrm{cA}$ & 1149 bA & $868 \mathrm{abA}$ & $818 \mathrm{abA}$ & 756 aA & $760 \mathrm{cB}$ & 618 bB & $570,5 \mathrm{abB}$ & $555 \mathrm{aB}$ & $533 \mathrm{aB}$ \\
\hline $\mathrm{Mg}$ & $253 \mathrm{dA}$ & $86 \mathrm{cA}$ & $51 \mathrm{bA}$ & $38 \mathrm{aA}$ & $36 \mathrm{aA}$ & $87 \mathrm{bB}$ & $46 \mathrm{aB}$ & $38 \mathrm{aB}$ & $38 \mathrm{aA}$ & $34 \mathrm{aA}$ \\
\hline $\mathrm{S}$ & - & - & - & - & - & - & - & - & - & - \\
\hline $\mathrm{Cu}$ & $0,11 \mathrm{aA}$ & $0,11 \mathrm{aA}$ & $0,11 \mathrm{aA}$ & $0,093 \mathrm{sA}$ & $0,08 \mathrm{aA}$ & $0,02 \mathrm{aB}$ & $0,03 \mathrm{aB}$ & $0,02 \mathrm{aB}$ & $0,01 \mathrm{aB}$ & $0,01 \mathrm{aB}$ \\
\hline $\mathrm{Fe}$ & $12,5 \mathrm{aA}$ & 11,7 aA & 11,2 aA & $11,1 \mathrm{aA}$ & $10,1 \mathrm{aA}$ & $17,3 \mathrm{bB}$ & $13,7 \mathrm{aB}$ & $14,7 \mathrm{aB}$ & $13,9 \mathrm{aB}$ & $13,2 \mathrm{aB}$ \\
\hline $\mathrm{Mn}$ & 16,9 aA & $11,0 \mathrm{aA}$ & $11,1 \mathrm{aA}$ & $11,1 \mathrm{aA}$ & $10,3 \mathrm{aA}$ & $11,5 \mathrm{aB}$ & $10,8 \mathrm{aA}$ & $10,3 \mathrm{aA}$ & $9,8 \mathrm{aA}$ & $9,7 \mathrm{aA}$ \\
\hline $\mathrm{Zn}$ & $23,3 \mathrm{dA}$ & $7,6 \mathrm{cA}$ & $7,6 \mathrm{cA}$ & $6,4 \mathrm{bA}$ & $3,0 \mathrm{aA}$ & $53,8 \mathrm{~dB}$ & $26,2 \mathrm{cB}$ & $21,4 \mathrm{cB}$ & $15,3 \mathrm{bB}$ & $4,8 \mathrm{aB}$ \\
\hline $\mathrm{Al}$ & 0 & 0 & 0 & 0 & 0 & 0 & 0 & 0 & 0 & 0 \\
\hline B & $0,52 \mathrm{cA}$ & $0,23 \mathrm{aA}$ & $0,21 \mathrm{aA}$ & $0,39 \mathrm{bA}$ & 0,15 aA & $0,35 \mathrm{cB}$ & $0,22 \mathrm{bA}$ & $0,17 \mathrm{aA}$ & $0,19 \mathrm{aB}$ & $0,20 \mathrm{abB}$ \\
\hline
\end{tabular}

valores de bases trocáveis $(\mathrm{K}, \mathrm{Ca}$ e $\mathrm{Mg}$ ) entre os macronutrientes e de $\mathrm{Cu}, \mathrm{Mn}$ e $\mathrm{B}$ entre os micronutrientes. Maiores teores de $\mathrm{Fe}$ e $\mathrm{Zn}$ foram encontrados em solos da formação psamófila-reptante (FPR). A concentração de nutrientes nos $10 \mathrm{~cm}$ superficiais seguiu a ordem decrescente: $\mathrm{Ca}>\mathrm{Mg}>\mathrm{K}>$ $\mathrm{P}>\mathrm{Zn}>\mathrm{Mn}>\mathrm{Fe}>\mathrm{B}>\mathrm{Cu}>\mathrm{Al}$ para a FP e de $\mathrm{Ca}>\mathrm{Mg}$ $>\mathrm{Zn}>\mathrm{P}>\mathrm{K}>\mathrm{Fe}>\mathrm{Mn}>\mathrm{B}>\mathrm{Cu}>\mathrm{Al}$ para a FPR.

Foi constatado gradiente decrescente de concentração dos nutrientes com a profundidade do solo (Tab. 1). A profundidade do solo foi acompanhada pelo decréscimo acentuado de macronutrientes, especialmente para o $\mathrm{Ca}$ e $\mathrm{Mg}$ na $F P$ e de $\mathrm{Zn}$ e o B entre os micronutrientes das duas formações. A variação na concentração de Fe só foi significativa no solo da FPR. Dentre os macronutrientes, somente o $\mathrm{P}$ apresentou índices elevados de fertilidade, enquanto que os demais apresentaram valores médios a baixos principalmente nas camadas superficiais (10 a $30 \mathrm{~cm}$ ) do solo da FPR. Os dados da Tab. 1 chamam a atenção pela ausência de $\mathrm{Al}$ nas diferentes camadas de solos das duas formações. Outras variáveis físico-químicas de solos são apresentadas na Tab. 2. O pH dos solos é alcalino e sem diferenças significativas entre as duas formações. Mesma

Tabela 2. Valores das variáveis físico-químicas da rizosfera de Passiflora mucronata Lam. e Canavalia rosea (Sw.) DC. na formação Palmae e psamófila-reptante, respectivamente, na restinga do Parque Estadual Paulo César Vinha, Guarapari, ES, Brasil. Nas linhas, as letras minúsculas comparam as médias dentro de profundidade e as letras maiúsculas comparam as médias entre as formações pelo teste de Duncan $(P<0,05 \%, \mathrm{n}=3) . \mathrm{Na}=$ sódio, $\mathrm{ISNa}=$ índice de saturação de sódio, $\mathrm{MO}=$ matéria orgânica, $\mathrm{SB}=$ soma de bases trocáveis, $\mathrm{CTC}=$ capacidade de troca catiônica total, $\mathrm{V}$ = percentagem de saturação de bases, $\mathrm{m}$ = índice de saturação de alumínio.

\begin{tabular}{|c|c|c|c|c|c|c|c|c|c|c|}
\hline \multirow[t]{3}{*}{ Variáveis } & \multicolumn{5}{|c|}{ Formação Palmae } & \multicolumn{5}{|c|}{ Formação psamófila-reptante } \\
\hline & \multicolumn{5}{|c|}{ Profundidade $(\mathrm{cm})$} & \multicolumn{5}{|c|}{ Profundidade $(\mathrm{cm})$} \\
\hline & 10 & 20 & 30 & 40 & 50 & 10 & 20 & 30 & 40 & 50 \\
\hline $\mathrm{pH}$ & $8,2 \mathrm{cA}$ & $8,4 \mathrm{cA}$ & 8,6 bcA & 8,7 bA & $8,9 \mathrm{bA}$ & 8,6 aA & $8,7 \mathrm{aA}$ & $8,7 \mathrm{aA}$ & $8,7 \mathrm{aA}$ & $8,8 \mathrm{aA}$ \\
\hline $\mathrm{Na}(\mathrm{mg} / \mathrm{kg})$ & $151 \mathrm{cA}$ & $128 \mathrm{acA}$ & $105 \mathrm{abA}$ & $101 \mathrm{abA}$ & $94 \mathrm{bA}$ & $119 \mathrm{bB}$ & $104 \mathrm{abB}$ & $95 \mathrm{aA}$ & $96 \mathrm{aA}$ & $95 \mathrm{aA}$ \\
\hline $\mathrm{ISNa}(\%)$ & $10 \mathrm{aA}$ & $15 \mathrm{aA}$ & $16 \mathrm{aA}$ & $17 \mathrm{aA}$ & $17 \mathrm{bA}$ & $18,4 \mathrm{aB}$ & $20,7 \mathrm{aB}$ & $20,5 \mathrm{aB}$ & $21,0 \mathrm{aB}$ & $21,7 \mathrm{aB}$ \\
\hline $\mathrm{MO}(\%)$ & $3,44 \mathrm{bA}$ & $1,01 \mathrm{aA}$ & $0,43 \mathrm{aA}$ & $0,18 \mathrm{aA}$ & $0,92 \mathrm{aA}$ & $2,01 \mathrm{bB}$ & $0,53 \mathrm{aB}$ & $0,25 \mathrm{aA}$ & $0,11 \mathrm{aA}$ & $0,04 \mathrm{aB}$ \\
\hline $\mathrm{SB}$ & $7,5 \mathrm{aA}$ & $3,8 \mathrm{bA}$ & $2,9 \mathrm{bA}$ & $2,7 \mathrm{bA}$ & $2,5 \mathrm{bA}$ & $2,83 \mathrm{bB}$ & $2,22 \mathrm{aB}$ & $2,02 \mathrm{aB}$ & $1,98 \mathrm{aB}$ & $1,91 \mathrm{aB}$ \\
\hline $\mathrm{CTC}\left(\mathrm{cmol}_{\mathrm{c}} / \mathrm{dm}^{3}\right)$ & $8,1 \mathrm{bA}$ & $4,3 \mathrm{aA}$ & $3,3 \mathrm{aA}$ & $3,1 \mathrm{aA}$ & $2,9 \mathrm{aA}$ & $3,2 \mathrm{bB}$ & $2,6 \mathrm{aB}$ & $2,4 \mathrm{aA}$ & $2,3 \mathrm{aB}$ & $2,3 \mathrm{aB}$ \\
\hline $\mathrm{V}(\%)$ & $93 \mathrm{cA}$ & $89 \mathrm{bA}$ & $87 \mathrm{abA}$ & $86 \mathrm{aA}$ & $86 \mathrm{aA}$ & $86 \mathrm{bB}$ & $83 \mathrm{aB}$ & $82 \mathrm{aB}$ & $82 \mathrm{aB}$ & $82 \mathrm{aB}$ \\
\hline $\mathrm{m}(\%)$ & 0 & 0 & 0 & 0 & 0 & 0 & 0 & 0 & 0 & 0 \\
\hline \multicolumn{11}{|c|}{ Textura $(\%)$} \\
\hline Areia Grossa & $78 \mathrm{aA}$ & $76 \mathrm{aA}$ & $78 \mathrm{aA}$ & $81 \mathrm{aA}$ & $81 \mathrm{aA}$ & $95 \mathrm{aB}$ & $96 \mathrm{aB}$ & $97 \mathrm{aB}$ & $95 \mathrm{aB}$ & $95 \mathrm{aB}$ \\
\hline Areia Fina & $21 \mathrm{aA}$ & $24 \mathrm{aA}$ & $21 \mathrm{aA}$ & $19 \mathrm{aA}$ & $18 \mathrm{aA}$ & $4,3 \mathrm{aB}$ & $4 \mathrm{aB}$ & $3 \mathrm{aB}$ & $4,6 \mathrm{aB}$ & $5 \mathrm{aB}$ \\
\hline Silte & 0 & 0 & 0 & 0 & 0 & 0 & 0 & 0 & 0 & 0 \\
\hline Argila & 0 & 0 & 0 & 0 & 0 & 0 & 0 & 0 & 0 & 0 \\
\hline
\end{tabular}


tendência ao relatado para os nutrientes minerais foi obtida em relação às bases trocáveis, $\mathrm{Na}, \mathrm{MO}$ e CTC que mostraram maiores valores no solo da FP. A maior fertilidade do solo da FP é confirmada pelo elevado teor de matéria orgânica (MO) em suas camadas superficiais.

Embora o teor de Na tenha sido maior na FP, o índice de saturação do $\mathrm{Na}$ (ISNa) foi maior na FPR mostrando a contribuição desse elemento no conteúdo total de cátions da formação sob maior influência da salinidade. $\mathrm{O} \mathrm{pH}$ foi alcalino e aumentou significativamente nas camadas mais profundas do solo. Os resultados das análises texturais (Tab. 2) demonstram que, em geral, os solos das duas formações foram predominantemente arenosos, de granulometria grossa com menores proporções de areia fina e isento de silte e argila. Solos da FPR foram constituídos basicamente por areia grossa e os solos da FP, por areia fina.

A análise da composição mineral foliar (Tab. 3) mostrou que as espécies possuem diferenças importantes quanto às exigências nutricionais. Com relação aos macronutrientes, $P$. mucronata seguiu a ordem decrescente $\mathrm{Ca}>\mathrm{N}>\mathrm{K}>\mathrm{S}>\mathrm{Mg}>\mathrm{P}$ enquanto que em $C$. rosea a proporção foi de $\mathrm{N}>\mathrm{Ca}>\mathrm{K}>\mathrm{Mg}>\mathrm{P}>\mathrm{S}$. Quanto aos micronutrientes, essa proporcionalidade foi de $\mathrm{Fe}>\mathrm{Zn}>$ $\mathrm{B}>\mathrm{Cu}>\mathrm{Mn}$ em $P$. mucronata e $\mathrm{Fe}>\mathrm{B}>\mathrm{Zn}>\mathrm{Cu}>\mathrm{Mn}$, em $C$. rosea. Diferenças significativas foram encontradas para o $\mathrm{P}, \mathrm{S}, \mathrm{Cu}$ e $\mathrm{Zn}$ cujas concentrações foram maiores em P. mucronata e de N, Fe e B, em C. rosea.

Nas duas espécies, ocorreram maiores acúmulos de macro e micronutrientes foliares em relação à concentração de nutrientes disponíveis no solo tendo como base os fatores de concentração de nutrientes (FC)

Tabela 3. Valores médios de macronutrientes $\left(\mathrm{g} \cdot \mathrm{kg}^{-1}\right)$ e micronutrientes $\left(\mathrm{mg} . \mathrm{kg}^{-1}\right)$ em folhas de Passiflora mucronata Lam. e Canavalia rosea (Sw.) DC. na restinga do Parque Estadual Paulo Cezar Vinha, Guarapari, ES, Brasil. Letras distintas referem-se às diferenças significativas encontradas pelo teste de Duncan a 5\% de significância $(P<0,05 \%, \mathrm{n}=5)$. Os valores que seguem as médias representam o desvio padrão. CV representa o coeficiente de variação.

\begin{tabular}{lccc}
\hline Nutrientes & P. mucronata & C. rosea & CV $(\%)$ \\
\hline$\left(\mathrm{g} . \mathrm{kg}^{-1}\right)$ & & & \\
$\mathrm{N}$ & $22,6 \pm 2,30 \mathrm{a}$ & $36,90 \pm 3,18 \mathrm{~b}$ & 9,3 \\
$\mathrm{P}$ & $2,1 \pm 0,4 \mathrm{a}$ & $1,52 \pm 0,38 \mathrm{~b}$ & 21,8 \\
$\mathrm{~K}$ & $13,1 \pm 3,17 \mathrm{~ns}$ & $11,00 \pm 3,23 \mathrm{~ns}$ & 26,5 \\
$\mathrm{Ca}$ & $28,0 \pm 6,08 \mathrm{~ns}$ & $21,90 \pm 2,54 \mathrm{~ns}$ & 18,6 \\
$\mathrm{Mg}$ & $3,6 \pm 1,02 \mathrm{~ns}$ & $4,50 \pm 0,31 \mathrm{~ns}$ & 18,5 \\
$\mathrm{~S}$ & $4,2 \pm 0,04 \mathrm{a}$ & $1,32 \pm 0,19 \mathrm{~b}$ & 5,0 \\
$\left(\mathrm{mg} \cdot \mathrm{kg}^{-1}\right)$ & & & \\
$\mathrm{Cu}$ & $3,72 \pm 1,90 \mathrm{a}$ & $0,88 \pm 0,63 \mathrm{~b}$ & 61,6 \\
$\mathrm{Fe}$ & $90 \pm 20,81 \mathrm{a}$ & $152 \pm 30,41 \mathrm{~b}$ & 21,5 \\
$\mathrm{Mn}$ & $0,01 \pm 0,0 \mathrm{~ns}$ & $0,01 \pm 0,0 \mathrm{~ns}$ & 0 \\
$\mathrm{Zn}$ & $51 \pm 15,42 \mathrm{a}$ & $13 \pm 0,81 \mathrm{~b}$ & 34,0 \\
$\mathrm{~B}$ & $24 \pm 5,58 \mathrm{a}$ & $38 \pm 5,09 \mathrm{~b}$ & 17,1 \\
\hline
\end{tabular}

apresentados na Tab. 4. Para a maioria dos nutrientes analisados, C. rosea apresentou maiores valores do fator FC do que $P$. mucronata. Em relação aos macronutrientes, $C$. rosea mostrou valores superiores do FC para o $\mathrm{Ca}, \mathrm{Mg}, \mathrm{Fe}, \mathrm{Cu}$ e B enquanto que $P$. mucronata se destacou pelos maiores do FC para o Pe Zn. As espécies não apresentaram diferenças significativas com o K. O valor do FC do $\mathrm{Zn}$ menor que 1 significa que a concentração foliar foi inferior à do solo.

Tabela 4. Fator de concentração* (FC) de Passiflora mucronata Lam. e Canavalia rosea (Sw.) DC. para os $10 \mathrm{~cm}$ superficiais de solo das formações Palmae e psamófila-reptante, respectivamente.

\begin{tabular}{lcrr}
\hline Nutrientes & P. mucronata & C. rosea & CV $(\%)$ \\
\hline $\mathrm{P}$ & $100 \pm 3,85 \mathrm{a}$ & $76 \pm 3,93 \mathrm{~b}$ & 4,4 \\
$\mathrm{~K}$ & $671 \pm 94,75 \mathrm{a}$ & $741 \pm 55,82 \mathrm{a}$ & 11,0 \\
$\mathrm{Ca}$ & $20 \pm 3,04 \mathrm{~b}$ & $33 \pm 1,17 \mathrm{a}$ & 8,5 \\
$\mathrm{Mg}$ & $31 \pm 7,43 \mathrm{~b}$ & $79 \pm 8,79 \mathrm{a}$ & 14,6 \\
$\mathrm{Zn}$ & $4,20 \pm 0,061 \mathrm{a}$ & $0,40 \pm 0,60 \mathrm{~b}$ & 18,5 \\
$\mathrm{Fe}$ & $7,59 \pm 0,23 \mathrm{~b}$ & $10,01 \pm 0,07 \mathrm{a}$ & 2,0 \\
$\mathrm{Cu}$ & $33 \pm 2,08 \mathrm{~b}$ & $38 \pm 1,83 \mathrm{a}$ & 5,5 \\
$\mathrm{~B}$ & $54 \pm 4,73 \mathrm{~b}$ & $149 \pm 12,31 \mathrm{a}$ & 9,2 \\
\hline
\end{tabular}

* FC foi determinado pela concentração foliar (ppm) dividida pela concentração encontrada nos $10 \mathrm{~cm}$ superficiais do solo (ppm).

\section{Discussão}

Os maiores teores de matéria orgânica e fertilidade do solo na FP também foram relatados em solos de regiões mais afastadas da praia na restinga de Marambaia no Rio de Janeiro (Menezes \& Araujo 2000). A fertilidade de solos pode interferir na estrutura da vegetação de restinga (Pereira 1990) e tem papel fundamental na manutenção de ecossistemas costeiros como os de Mata Atlântica, pois detém a reserva de nutrientes $(\mathrm{N}, \mathrm{P}, \mathrm{S}$, $\mathrm{Ca}, \mathrm{Mg}, \mathrm{K}$ e $\mathrm{Na}$ ), melhora a capacidade de retenção de água, influencia o $\mathrm{pH}$, eleva a capacidade de troca catiônica (CTC) evitando, assim, a lixiviação de cátions (Zech et al. 1997). Além das diferenças nutricionais entre as formações analisadas, um gradiente de fertilidade foi verificado no perfil do solo onde as camadas mais superficiais mostraram maiores teores de macronutrientes e de MO, especialmente na formação FP.

Outra resposta associada à fertilidade de solos está na disponibilidade do P (Marschner 1995) na restinga de Guarapari. Segundo Hay \& Lacerda (1984), a entrada de $\mathrm{P}$ nos ecossistemas de restinga se dá, principalmente, através da deposição de salsugem. Este fato poderia explicar a maior presença de P nos solos da FPR e da FP e a sua baixa ocorrência em locais mais distantes da praia (Pereira 1990a).

Embora a saturação por Al tenha associação na composição florística nas formações de arbóreas em 
restingas de outras localidades (Moraes et al. 1999; Guedes et al. 2006) e do PEPCV (Fabris 1995), isso não parece se aplicar nas formações herbáceas da restinga de Guarapari devido à ausência de $\mathrm{Al}$ em solos da $\mathrm{FP}$ e FPR. No entanto, baixas concentrações de $\mathrm{Al}$ foram detectadas na FP da restinga de Marambaia, Rio de Janeiro (Menezes \& Araujo 2000). Um gradiente de Al no sentido praia-continente parece ocorrer em ambientes de restinga devido aos níveis mais elevados desse elemento na formação aberta e de mata seca na restinga de Guarapari (Fabris 1995). A ausência de Al na FP e FPR pode estar associada ao $\mathrm{pH}$ do solo que, quando alcalino, torna o $\mathrm{Al}$ indisponível provocando deficiência de micronutrientes, elevação dos percentuais de V e CTC e perda de N pela volatilização (Tomé Jr. 1997). Além disso, $\mathrm{pH}$ acima de 7,5 é indicativo de excesso de sais no solo como demonstrado pelas concentrações mais elevadas de Ca nas camadas superficiais do solo da FPR. Dessa forma, a maior deficiência de micronutrientes em solos da FPR pode estar relacionada ao maior $\mathrm{pH}$. No entanto, o pH alcalino encontrado nessa localidade não foi acompanhado pelo aumento dos valores de V e CTC. Tomé Jr. (1997) afirma que estas situações podem ocorrer em solos arenosos mostrando a importância da granulometria na constituição química do solo.

A redução da concentração de $\mathrm{Ca}$ com o aumento da profundidade do solo não foi acompanhada pela diminuição do $\mathrm{pH}$, mas sim pela MO. Segundo Santos \& Camargo (1999) maiores teores de MO promove o aumento da CTC do solo facilitando a troca de cátions essenciais ao crescimento vegetal ( $\mathrm{Ca}, \mathrm{Mg}, \mathrm{Cu}, \mathrm{Zn}$ e $\mathrm{K}$ ) por íons $\mathrm{H}^{+}$. Esse mecanismo de troca iônica poderia ter causado a redução do $\mathrm{pH}$ nas camadas superficiais do solo onde se verificou maior teor de MO e, conseqüentemente, maior capacidade de retenção de íons $\mathrm{H}^{+}$. Comportamento similar foi verificado em solos de região amazônica e do cerrado onde a redução da MO foi acompanhada por maiores valores de $\mathrm{pH}$ nas camadas mais profundas de seus solos (Demattê \& Demattê 1993). Entretanto, esses autores verificaram que maiores conteúdos de MO se deu em solos com maior fração de argila, demonstrando a importância do aspecto textural na distribuição da MO do solo.

As maiores frações de areia fina na FP da restinga de Guarapari podem ter contribuído para a maior fertilidade dos solos associada à topografia do relevo, determinante no posicionamento de arbóreas em ecossistema de florestas tropicais (Espírito Santo et al. 2002; Souza et al. 2003; Carvalho et al. 2005; Yimer et al. 2006; Rodrigues et al. 2007). Sedimentos arenosos de granulometria média a grossa dominam as restingas capixabas (Pereira 1990b, Thomaz 1991). As restingas do sudeste foram formadas em dois períodos geológicos, originando dois cordões arenosos (Willwock et al. 2005). Portanto, a localização da FP após a FPR em Guarapari sugere a possibilidade do efeito eólico em promover maior depósito de areia fina em locais mais distantes do mar.

A participação dos fatores edáficos na composição florística é reconhecida para alguns ecossistemas costeiros devido ao impacto da areia e da salsuagem limitando o porte das plantas mais próximas à praia (Pires et al. 2006). O maior índice de salinidade (ISNa) no solo da FPR confirma sua propriedade halomórfica e demonstra a importância do Na no somatório de cátions (SB). Devido à sua ação instabilizadora sobre a estrutura do solo, o Na afeta a formação de agregados de MO (Tomé JR 1997).

Quanto à proporção e concentração interna de nutrientes foliares, diferenças mais expressivas foram observadas com o $\mathrm{Ca}$ mais representativo em P. mucronata e o N, em C. rosea. Esses resultados não podem ser atribuídos, exclusivamente, à composição nutricional do solo, pois o menor teor de $\mathrm{MO}$ na rizosfera de $C$. rosea não refletiu em menores concentrações de $\mathrm{N}$ foliar. A localização de $C$. rosea na FPR caracterizada pela baixa fertilidade sugere que maior acúmulo de $\mathrm{N}$ foliar tenha ocorrido pela fixação biológica através de seus nódulos radiculares (Chen et al. 2000). Os elevados níveis de $\mathrm{Fe}$ nas folhas de $C$. rosea sustentam esta hipótese, uma vez que a absorção desse elemento aumenta em leguminosas que crescem em solos alcalinos e com maiores teores de Fe (Porter \& Lawlor 1991). Deve-se ressaltar também que o Fe faz parte da estrutura molecular das enzimas fixadoras do nitrogênio atmosférico e do transportador ferrodoxina integrante dos processos metabólicos da redução do nitrogênio (Marschner 1995). As deficiências nutricionais e baixas concentrações de $\mathrm{Al}$ e de prótons $\mathrm{H}^{+}$no solo, como verificado na FPR, estimula a nodulação (Marschner 1995) e leva à maior absorção de nutrientes minerais, verificado na maior concentração de $\mathrm{Ca}, \mathrm{Mg}, \mathrm{Fe}, \mathrm{Cu}$ e B nas folhas de C. rosea. A importância das associações simbióticas em ambiente de restinga tem sido confirmada pela ampla representatividade de plantas leguminosas nesse ambiente (Pereira \& Araujo 2000).

As propriedades físico-químicas do solo representam um dos fatores ambientais mais importantes na distribuição espacial e na estrutura de florestas tropicais (Cuzzuol \& Campos 2001; Espírito Santo et al. 2002; Souza et al. 2003; Carvalho et al. 2005; Bernini et al. 2006; Yimer et al. 2006; Rodrigues et al. 2007). Os resultados obtidos na restinga de Guarapari sugerem que as características pedológicas têm papel chave no estabelecimento de $C$. rosea na FPR de P. mucronata na FP. Uma análise da nutrição mineral e da salinidade no 
estabelecimento de plântulas pode gerar informações mais contundentes sobre a dinâmica de distribuição espacial das espécies em ambiente de restinga.

\section{Agradecimentos}

Ao Instituto Capixaba de Pesquisa e Extensão Rural (Incaper), pelas análises de solo e do material biológico. O primeiro autor agradece à Petrobrás, pela concessão de bolsa; aos professores MSc. Oberdan José Pereira e Dr. Luiz Fernando Tavares de Menezes, pela revisão e sugestões.

\section{Referências bibliográficas}

Assis, A.M.; Thomaz, L.D. \& Pereira, O.J. 2004. Florística de um trecho de floresta de restinga no município de Guarapari, Espírito Santo, Brasil. Acta Botanica Brasilica 18: 191-201.

Araujo, D.S.D. 1992. Vegetation types of sandy coastal plains of tropical Brazil: a first aproximation. In: U. Seeliger (coord.). Coastal plant communities of Latin America. New York, Academic Press.

Bataglia, O.C.; Furlani, A.M.C.; Teixeira, J.P.F.; Furlani, P.R. \& Gallo, J.R. 1983. Métodos de análise química de plantas. Instituto Agronômico de Campinas. Boletim Técnico 78.

Bernini, E.; Silva, M.A.B.; Carmo, T.M.S.C. \& Cuzzuol, G.R.F.C. 2006. Composição química do sedimento e de folhas das espécies do manguezal do estuário do Rio São Mateus, Espírito Santo, Brasil. Revista Brasileira de Botânica 29: 689-699.

Carvalho, D.A.; Oliveira Filho, A.T.; Van Den Berg, E.; Fontes, M.A.L.; Vilela, E.A.; Marques, J.J.G. \& Carvalho, W.A.C. 2005. Variações florísticas e estruturais do componente arbóreo de uma floresta ombrófila alto-montana às margens do rio Grande, Bocaina de Minas, MG, Brasil. Acta Botanica Brasilica 19: 91-109.

Chen, W.V.; Lee, T.M.; Lan, C.C. \& Cheng, C.P. 2000. Characterization of halotolerant rhizobia isolated from root nodules of Canavalia rosea from seaside areas. Fems Microbiology Ecology 34: 9-16.

Cogliatti-Carvalho, L.; De Freitas, A.F.N.; Rocha, C.F.D. \& Sluys, M.V.N. 2001. Variação na estrutura e na composição de Bromeliaceae em cinco zonas de restinga no Parque Nacional da Restinga de Jurubatiba, Macaé, RJ. Revista Brasileira de Botânica 24: 1-9.

Costa, S.N.; Salgado, J.S. \& Costa, A.F.S. 2005. Solos, nutrição e adubação do maracujazeiro. In: Tecnologias para a produção de maracujá. Vitória, INCAPER.

Cuzzuol, G.R.F. \& Campos, A. 2001. Aspectos nutricionais na vegetação de manguezal do estuário do rio Mucuri, Bahia, Brasil. Revista Brasileira de Botânica 24: 227-234.

Demattê, J.L.I. \& Demattê, J.A.M. 2003. Comparações entre as propriedades químicas de solos das regiões da floresta amazônica e do cerrado do Brasil central. Scientia Agrícola 50: 272-286.

Embrapa. 1997. Centro nacional de pesquisas de solos. Manual de Métodos de Análises de Solo. Rio de Janeiro, EMBRAPACNPS.
Espírito-Santo, F.D.B.; Oliveira Filho, A.T.; Machado, E.L.M. \& Souza, J.S. 2002. Variáveis ambientais e a distribuição de espécies arbóreas em um remanescente de floresta estacional semidecídua montana no campus da Universidade Federal de Lavras, MG. Acta Botanica Brasilica 16: 331-356.

Fabris, L.C. 1995. Composição florística e fitossociológica de uma faixa de floresta arenosa litorânea do Parque Estadual de Setiba, Município de Guarapari, ES. Dissertação de Mestrado. Rio Claro, Universidade Estadual Paulista.

Fabris, L.C. \& César, O. 1996. Estudos florísticos em uma mata litorânea no sul do estado do Espírito Santo. Boletim do Museu de Biologia Mello Leitão 5: 15-46.

Fontes, M.A.L. \& Marques, J.J.G. 2002. Variáveis ambientais e a distribuição de espécies arbóreas em um remanescente de floresta estacional semidecídua montana no campus da Universidade Federal de Lavras, MG. Acta Botanica Brasilica 16: 331-356.

Guedes, D.; Barbosa, L.M. \& Martins, S.E. 2006. Composição florística e estrutura fitossociológica de dois fragmentos de floresta de restinga no Município de Bertioga, SP, Brasil. Acta Botanica Brasilica 20: 299-311.

Hay, J.D. \& Lacerda, L.D. 1984. Ciclagens de nutrientes no ecossistema restinga. Pp. 459-475. In: L.D. Lacerda et al. (orgs.). Restingas: origens, estruturas e processos. Niterói, CEUFF.

Henriques, R.P.B.; Araujo, D.S.D. \& Hay, J.D. 1986. Descrição e classificação dos tipos de vegetação da restinga de Carapebus, Rio de Janeiro. Revista Brasileira de Botânica 9: 173-189.

Jefferies, R.L. \& Davy, A.J. 1979. Ecological processes in coastal environments. Oxford, Blackwell Scientific Publications.

Marschner, H. 1995. Mineral Nutrition of Higher Plants. London, Academic Press.

Menezes, L.F.T. \& Araujo, D.S.D. 1999. Estrutura de duas formações vegetais do cordão externo da restinga de Marambaia, RJ. Acta Botanica Brasilica 13: 223-235.

Menezes, L.F.T. \& Araujo, D.S.D. 2000. Variação da biomassa aérea de Allagoptera arenaria (Gomes) O. Kuntze (Arecaceae) em uma comunidade arbustiva de Palmae na restinga de Marambaia, RJ. Revista Brasileira de Biologia 60: 147-157.

Moraes, R.M.; Delitti, W.B.C. \& Struffaldi-De-Vuono, Y. 1999. Litter fall and litter nureant content two Brazilian Tropical Forest. Revista Brasileira de Botânica 50: 163-173.

Pereira, O.J. 1990a. Caracterização fisionômica da restinga de Setiba, Guarapari-ES. Pp. 207-220. In: $2^{\circ}$ Simpósio de ecossistemas da costa sul e sudeste brasileira: estrutura, função e manejo. Águas de Lindóia, ACIESP.

Pereira, O.J. 1990b. Levantamento florístico fitossociológico de uma área de restinga do Estado do Espírito Santo. Dissertação de Mestrado. Rio de Janeiro, Universidade Federal do Rio de Janeiro.

Pereira, O.J. \& Araujo, D.S.D. 2000. Análise florística das restingas dos estados do Espírito Santo e Rio de Janeiro. Pp. 207-219. In: L.D. Lacerda (org.). Ecologia de Restingas e Lagoas Costeiras. Rio de Janeiro, NUPEM/UFRJ.

Pires, L.A.; Britez, R.M.; Martel, G. \& Pagano, S.N. 2006. Produção, acúmulo e decomposição da serapilheira em uma restinga da ilha do Mel, Paranaguá, PR, Brasil. Acta Botanica Brasilica 20: 173-184. 
Porter, J.R. \& Lawlor, D.W. 1991. Plant Growth: Interactions with nutrition and environment. New York, Cambridge University Press.

Reinert, F.; Roberts, A.; Wilson, J.M.; Ribas, L.; Cardinot, G. \& Griffith, H. 1997. Gradation in nutrient composition and photosynthetic pathway across the restinga vegeration of Brazil. Acta Botanica Brasilica 10: 135-142.

Rodrigues, L.A.; Carvalho, D.A.; Oliveira Filho, A.T. \& Curi, N. 2007. Efeitos de solos e topografia sobre a distribuição de espécies arbóreas em um fragmento de floresta estacional semidecidual, em Luminárias, MG. Revista Árvore 31: 25-35.

Santos, G.A. \& Camargo, F.A.O. 1999. Fundamentos da matéria orgânica do solo: ecossistemas tropicais e subtropicais. Porto Alegre, Gênese.

Scherer, A.; Maraschin-Silva, F. \& Moura Baptista, L.R. 2005. Florística e estrutura do componente arbóreo de matas de Restinga arenosa no Parque Estadual de Itapuã, RS, Brasil. Acta Botanica Brasilica 19: 717-726.

Suguio, K.; Martin, L. 1990. Geomorfologia das restingas. Pp. 185-205. In: $2^{\circ}$ Simposio de ecossistema da costa Sul e Sudeste brasileira: estrutura, função e manejo. Águas de Lindóia, ACIESP.

Tomé Jr., J.B. 1997. Manual para interpretação de análise de solo. Guaíba, Editora Agropecuária.
Thomaz, L.D. \& Monteiro, R. 1991. Distribuição e diversidade na vegetação halófila-psamófila no litoral do Espírito Santo. Dissertação de Mestrado. Rio Claro, Universidade Estadual Paulista.

Villela, D.M.; Nascimento, M.T.; Mazurec, A.P.; Gonçalves, G.M. \& Rezende, C.E. 2001. Soil Chemical properties under Eucalyptus citiodora plantations of different ages after a 9year period of abandonment in União Biological Reserve, Rio de Janeiro State, Brazil. In: CR-ROOM of the $3^{\text {rd }}$ international conference on land degradation and meeting of the IUSS subcommission $\mathbf{C}$ : soil and water conservation. Rio de Janeiro, Brazil.

Willwock, J.A.; Lessa, G.C.; Suguio, K.; Angulo, R.J. \& Dillenburg, S.R. 2005. Geologia e geomorfologia de regiões costeiras. Pp. 382. In: C.R.G. Souza; K. Suguio; A.M.S.O. Oliveira \& P.E. Oliveira. Quaternário do Brasil. Ribeirão Preto, Holos.

Yimer, F.; Ledin, S. \& Abdelkadir, A. 2006. Soil property variations in relation to topographic aspect and vegetation community in the south-eastern highlands of Ethiopia. Forest Ecology and Management 232: 90-99.

Zech, W.; Senesi, N.; Guggenberger, G.; Kaiser, K.; Lehmann, J.; Miano, T.M.; Miltner, A. \& Schroth, G. 1997. Factors controlling humification and mineralization of soil organic matter in the tropics. Geoderma 79: 117-161. 